\title{
Maximum relaxation rate of the diaphragm during weaning from mechanical ventilation
}

\author{
John C Goldstone, Malcolm Green, John Moxham
}

\begin{abstract}
Background - The maximum relaxation rate (MRR; percentage fall in pressure/ $10 \mathrm{~ms}$ ) of oesophageal (POES) and transdiaphragmatic (PDI) pressure slows under conditions of loaded breathing, and has been measured previously in normal subjects. MRR has not been measured in intubated patients weaning from mechanical ventilation.

Methods - Five postoperative patients who were expected to wean and nine patients who had previously failed were studied. POES and PDI MRR, peak oesophageal pressure during spontaneous breathing, maximum oesophageal pressure, and the inspiratory duty cycle were measured at rest during mechanical ventilation, in the first two minutes of spontaneous breathing, and after reventilation in those patients who failed, or before extubation in those patients who succeeded.
\end{abstract}

Results - At rest PoEs MRR in intubated patients had a range of 5:6-11 and PDI MRR 6.9-10.0, with a coefficient of variation of $9.9 \%$ and $7 \cdot 3 \%$ respectively. POES and PDI MRR were similar before and after extubation in five postoperative patients, and PoEs MRR was reflected by endotracheal MRR measured at the airway. In five patients who failed to wean POES and PDI MRR slowed by $47 \%$ and $44 \%$, and fully recovered after 10 minutes reventilation. In four patients who were successfully weaned MRR was unchanged during spontaneous breathing. At the time when MRR decreased, the respiratory muscles were heavily loaded in relation to their strength.

Conclusions - Weaning failure occurs when the applied load exceeds the capacity of the respiratory muscles, and this is associated with a slowing of respiratory muscle MRR.

(Thorax 1994;49:54-60)

The ability to sustain ventilation is dependent on central nervous system drive, the strength of the respiratory muscles, and the load applied to them. Available data suggest that central drive to the respiratory muscles is elevated in ventilatory failure,${ }^{1}$ and in patients who are difficult to wean those who eventually fail have a greater drive than patients who succeed. ${ }^{2}$ Attention has therefore focused on respiratory muscle function where investigators have sought to establish the importance of respiratory muscle fatigue during weaning.
Respiratory muscle fatigue has been defined as an acute failure to generate the required or expected force or velocity which is reversible with rest. ${ }^{3}$ It can be induced in normal subjects during loaded breathing through an inspiratory resistance. ${ }^{4}$ The degree and duration of loading needed to produce fatigue has been extensively investigated ${ }^{5}$ and can be numerically quantified to predict when force loss will occur. $^{6}$ By their nature, these studies have focused on the end point of loaded breathing, usually failure to achieve target pressures. Clinically, most attention is directed at observing the performance of patients at the beginning of a trial of spontaneous breathing in order to predict eventual weaning failure.

Under conditions of heavy load the rate of contraction and relaxation of muscle slows. The relaxation rate of the respiratory muscles has been measured from the rate of change of pressure after maximal or submaximal contractions of the diaphragm, either from sustained contractions ${ }^{7}$ or after brief inspiratory sniffs $^{8}$ or gasps. ${ }^{9}$ Pressure has been measured in the oesophagus (PoEs), across the diaphragm (PDI), or in the upper airway. ${ }^{8}$ As the rate of relaxation depends in part on the amplitude of the waveform, relaxation rate is expressed as the percentage fall in pressure over $10 \mathrm{~ms}$ (MRR). ${ }^{10}$ From investigations in normal subjects, changes in MRR occur early during severely loaded breathing preceding failure to generate the required pressure, reach a plateau value which is sustained during the loaded period, and recover rapidly after rest. ${ }^{11} \mathrm{Al}$ though MRR has been used to explore the importance of severe loading of the respiratory muscles in the laboratory setting, it has not been measured previously in ventilated patients submitted to weaning trials.

The aim of this study was to measure MRR in intubated patients in the intensive care unit, to assess the practical importance of intubation on the measurement of relaxation rate, and to measure MRR in patients weaning from mechanical ventilation.

\section{Methods}

The study was approved by the hospital ethical committee of King's College Hospital and all normal subjects and patients gave informed consent. We studied four normal men (age range 29-41 years) and 14 intubated patients (seven men, age range 41-78 years). Of these patients, five were recovering from routine surgery and nine had received mechanical ventilation for a number of days or weeks and were currently weaning. Patients in the weaning group were included when they were able 
to breathe unassisted for a minimum of 15 minutes without the development of hypercapnia, required an inspired oxygen fraction of less than $50 \%$, and were awake and able to follow verbal commands. Patients were excluded if febrile or unable to maintain oxygenation.

Pressure was measured in the oesophagus (POES) and across the diaphragm (PDI) with $10 \mathrm{~cm}$ balloon catheters ( $\mathrm{P}$ Morgan, Rainham, Kent, UK) connected to Validyne MP-45 pressure transducers with a range of $\pm 300 \mathrm{~cm} \mathrm{H}_{2} \mathrm{O}$ (Validyne Co, Northridge, California, USA). Transdiaphragmatic pressure (PDI) was derived electronically by subtraction of PoEs from PGA. In normal subjects upper airway pressure was also simultaneously recorded from a side arm port at the level of the mouthpiece.

The oesophageal and gastric balloons were passed through the nose after topical anaesthesia with $4 \%$ lignocaine to the nose and nasopharynx to a distance of $70 \mathrm{~cm}$ from the anterior nares. The oesophageal balloon was then withdrawn from the stomach into the oesophagus and the position adjusted to reduce the cardiac artefact. ${ }^{12}$

Intubated patients cannot sniff naturally because their upper airway is bypassed by the endotracheal or tracheostomy tube. We therefore attached a device to the endotracheal tube which limited flow in a similar manner to that observed in studies of the upper airway during freely performed sniffs. ${ }^{13}$ The device was a simple flap valve which occluded flow during inspiration, thereby allowing the patient to generate a "sniff-like" inspiratory waveform.

Normal subjects were studied in order to show that MRR measured from inspiratory efforts through the flap valve slowed during loaded ventilation, and to investigate the transmission of waveforms from the thorax to the upper airway using the device. In the studies in normal subjects pressure was recorded onto an ink jet polygraph (Mingograf 800 , Siemens Elema) and measured by hand. In order to measure pressures and MRR at the bedside we developed a mobile computer based system. Analogue signals were fed via an eight-channel A-D converter to a personal computer (BBC Master, Acorn Electronics, UK). A program was developed to display pressure signals in real time on the screen. Each pressure signal was differentiated and algorithms written to measure the maximum amplitude of the pressure waveform, and the maximum rate of change of pressure (dPoEs/dt). MRR was obtained by dividing dPoEs/dt by PoEs and expressed as $\%$ fall in pressure $/ 10 \mathrm{~ms}$. Data were then stored on floppy disk for subsequent review.

Sniffs were assessed in terms of acceptability in a manner described previously in laboratory subjects, namely that peak pressure should have a duration of less than $50 \mathrm{~ms}$, the decay of pressure should occur smoothly without oscillations, and the duration of the inspiratory effort should not exceed $500 \mathrm{~ms}^{8}$

Chest and abdominal wall position was monitored by a respiratory inductive plethys- mograph, RIP (Respitrace Inc, Ardsley, New York, USA). Sniffs were performed from the relaxed end expiratory point, as judged from the oesophageal pressure and RIP signal.

Oesophageal pressure was measured during representative periods of quiet breathing. $\mathrm{T} I /$ TTOT was calculated from the inspiratory pressure signal. TI was measured from the onset of the negative oesophageal pressure until the pressure signal returned to the baseline measurement. Tidal volume was measured by an in-line mechanical spirometer (Wright's respirometer). Maximum pressure was recorded by instructing the patient to perform inspiratory efforts through the flap valve in a similar manner to that during a maximal, freely performed sniff. Patients received verbal encouragement, visual oscilloscope feedback, and made three maximum efforts. The maximum oesophageal pressure generated during this manoeuvre was recorded.

All patients undergoing weaning trials were assessed independently by the medical team responsible for their care, and respiratory rate, heart rate, abdominal paradox, and respiratory alternans were recorded during spontaneous breathing. These measurements were recorded without knowledge of simultaneous recordings of pressure, MRR, and duty cycle. A clinical impression of respiratory distress was recorded when the patients complained of shortness of breath, were unable to cooperate because of restlessness, or when they requested reventilation.

Mean data, regression analysis, paired $t$ tests, analysis of variance, and correlation coefficients were obtained from the Minitab statistical program (Minitab Inc, State College, Pennsylvania, USA).

\section{PROTOCOL \\ Normal subjects}

The normal subjects were laboratory workers familiar with the measurement techniques who had experience in performing loaded breathing to exhaustion. Maximum static inspiratory oesophageal pressure was measured for all subjects. The subjects sat in an upright chair in front of a storage oscilloscope. Oesophageal pressure was displayed on the screen, and a second trace was adjusted so that it represented $80 \%$ of the maximum inspiratory pressure. The subject performed 10 rapid inspiratory "sniff-like" efforts through the flap valve, which was held in the mouth with the nose clipped, with rest between each effort. Each subject then breathed through an alinear resistance, achieving the $80 \%$ target with a square wave pressure during each breath, while expiration was unloaded. The subjects received verbal encouragement and visual feedback during each study. The end point occurred when three consecutive breaths failed to meet the target pressure. The subjects then performed brief maximal inspiratory efforts through the flow limiting valve at a rate of one sniff every 10 seconds over the ensuing five minutes. Further sniffs were recorded at 10 minutes after loading. 
Intubated patients

Five postoperative patients were studied after 4-6 hours of ventilatory assistance before and after extubation. All patients had coronary revascularisation surgery and ventilatory assistance was withdrawn when the patient had regained consciousness and was haemodynamically stable.

The nine patients studied during trials of weaning had been mechanically ventilated for more than seven days. All patients were orientated and alert. Previous attempts at weaning had resulted in hypercapnic respiratory failure in all patients. Entry into the study occurred when (1) the patients had recovered from the critical event that precipitated admission to the intensive care unit and were clinically stable; (2) oxygen requirements were met with an $\mathrm{FIO}_{2}$ of $\leqslant 0.50$ and were stable; and (3) all were able to tolerate discontinuation from mechanical ventilation for at least 0.5 hour as part of their treatment in the intensive care unit.

Weaning was initiated and supervised by the medical team responsible for the care of the patients. The patients were studied reclining in bed at $45^{\circ}$ and this position was maintained during the weaning trial. Ventilation over the preceding eight hours had been by controlled minute ventilation, achieved by increasing the respiratory rate until the patient was not triggering the ventilator for more than one breath per minute.

The patients were connected to the ventilator via a three way tap in such a manner that they could be switched to the flap valve intermittently to enable them to perform brief "sniff-like" inspiratory efforts whilst still receiving mechanical ventilation. In addition to the patients included in this study, five patients were unable to perform sniffs which were suitable for analysis and were therefore not studied during weaning from mechanical ventilation. All patients were able to perform sniff manoeuvres after initial training which did not exceed 30 minutes, and following rest it was then possible to record baseline oesophageal and transdiaphragmatic sniff pressures in order to estimate POES and PDI MRR before weaning.

Following baseline measurements made intermittently during mechanical ventilation, the patients were attached to a t-piece breathing circuit, breathing oxygen enriched humidified air. During the next 1-2 minutes oesophageal pressure, tidal volume, and the duty cycle were measured for each breath. These measurements were also recorded at the end of the weaning trial immediately before reinstitution of mechanical ventilation or at eight hours in those weaning successfully.

Cardiovascular and respiratory vital signs were monitored by the medical and nursing staff. Criteria for reventilation were tachypnoea (respiratory rate greater than 35 breaths/ min), a fall in tidal volume (by greater than $20 \%$ of initial volume), a high minute ventilation (greater than $20 \mathrm{l} / \mathrm{min}$ ), deterioration of vital signs or distress as assessed by the clinical staff.

\section{Results}

NORMAL SUBJECTS

Using the flow limiting valve, mean (SE) MRR ( $\%$ fall in pressure $/ 10 \mathrm{~ms}$ ) for the four normal subjects when fresh was $9.41(0.3)$ for PoEs and $8 \cdot 24(0 \cdot 6)$ for PDI (fig 1). MRR declined in all subjects after inspiratory loading. Thirty seconds after failure to continue loaded breathing MRR was reduced by $25 \%$ of the control value (range 23-29\%) for POES and by $26 \%$ (range $20-30 \%$ ) for PDI. At one minute after recovery MRR was significantly reduced from the control value for POES (paired $t$ test,
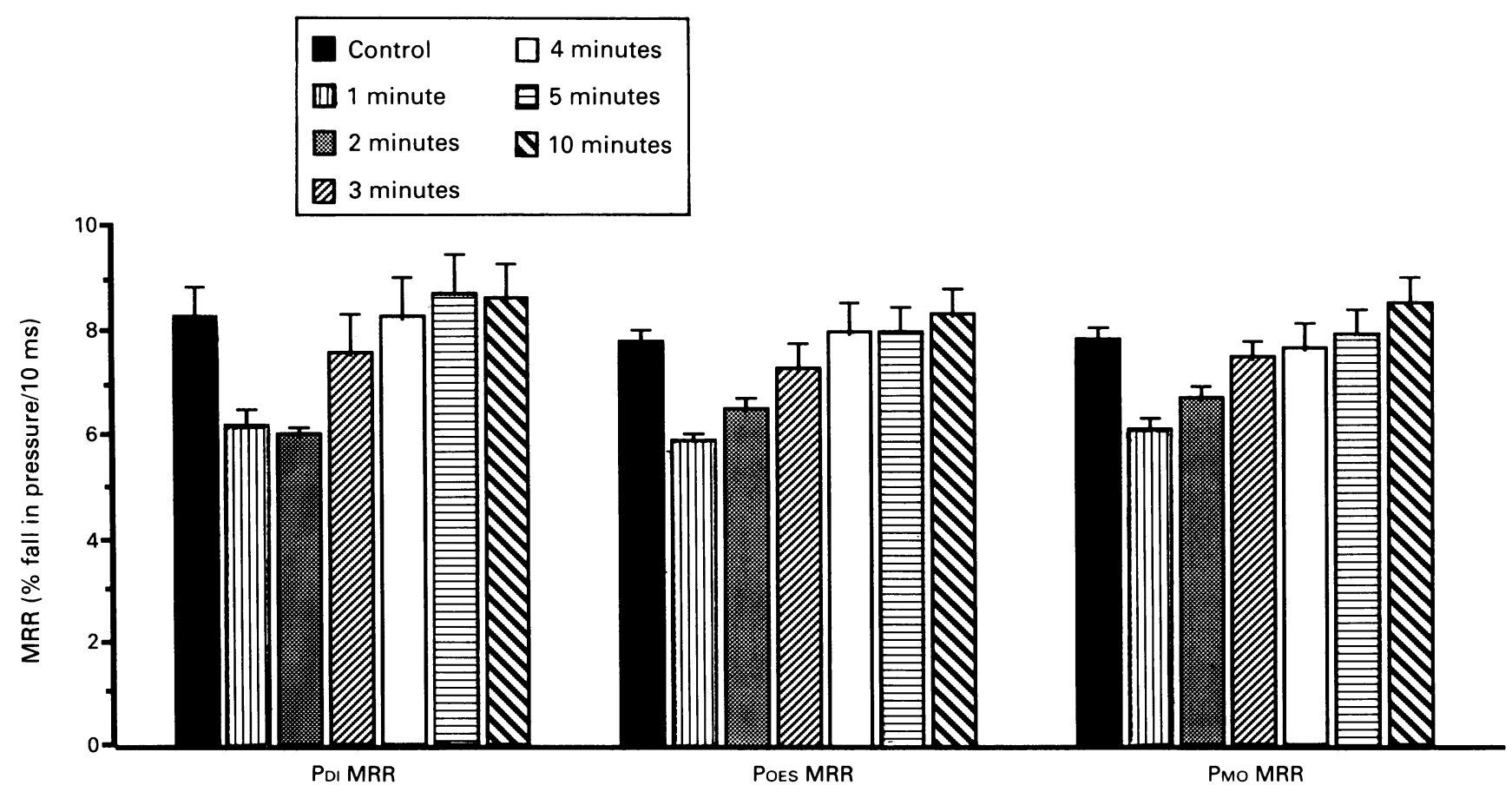

Figure 1 Diaphragmatic (PDI), oesophageal (POES), and mouth (PMO) MRR measured before (control) and at one, two, three, four, five, and 10 minutes after inspiratory resistive breathing in four normal subjects. Results are expressed as the mean and standard error. 
$\mathrm{p}<0.001)$ and PDI $(\mathrm{p}<0.001)$. MRR was not different from the control value by the fourth minute for PoEs and PDI (fig 1).

MRR measured at the mouth with the flow limiting valve was closely similar to MRR measured in the oesophagus (fig 1). Before, during, and after recovery from fatigue $M R R$ at the mouth closely followed MRR in the oesophagus. The correlation coefficient $(r)$ of POES and PMO MRR was 0.980, p $<0.001$. Regression analysis showed that POES $\operatorname{MRR}=-0.908+1.1$ PMO $\operatorname{MRR}\left(R^{2}=0.952 \%\right.$, $\mathrm{SEE}=0.23$ ).

\section{INTUBATED PATIENTS}

Descriptive data are given for the patients in table 1. Of those patients who were ventilated for more than seven days, four were able to be weaned and continued to breathe spontaneously after the eight hour study period and were then extubated. In the following 48 hours no extubated patient was reventilated.

In five routine postoperative patients intubated POES MRR agreed closely with that found during a freely performed sniff after extubation (mean intubated POES MRR 8.63, extubated MRR 8.22). Intubated PDI MRR also agreed closely with MRR when extubated (intubated MRR 8.41, extubated MRR 8.23). The mean difference (intubated-extubated) for all efforts and subjects for POES MRR was 0.38 (range -0.05 to $1 \cdot 1$ ) and for PDI MRR was 0.48 (range -0.8 to $1 \cdot 1$ ). Simultaneous measurements of POES MRR and MRR measured within the endotracheal tube (PET MRR) agreed closely, with a mean difference (PoEs MRR - PET MRR) between the two sites of measurement of 0.4 (95\% CI -1.36 to $2 \cdot 16)$.

During mechanical ventilation MRR was estimated from triplicate inspiratory efforts. Mean Poes MRR of all 14 patients was 8.53 (range 5.6-11.0). The coefficient of variation of mean POES MRR was $9.9 \%$ (range 3.4 20.4\%). Mean PDI MRR was 8.54 (range 6.9. 10) with a mean coefficient of variation of $7 \cdot 3 \%$ (range $2 \cdot 4-22 \%$ ). In four patients who were successfully weaned we measured the within day variability of POES MRR and PDI MRR by measuring efforts on three occasions within an eight hour period. The mean coefficient of variation for POES MRR for the four subjects

Table 1 Descriptive data of nine patients (1-9) submitted to a trial of weaning and five postoperative cardiac patients $(10-14)$

\begin{tabular}{llllll}
\hline Patient & Diagnosis & Age & Sex & $\begin{array}{l}\text { Days ventilated } \\
\text { before weaning }\end{array}$ & $\begin{array}{l}\text { Outcome of weaning } \\
\text { trial }\end{array}$ \\
\hline 1 & COPD; post CVG & 73 & M & 77 & Failed \\
2 & Muscular dystrophy & 33 & M & 60 & Failed \\
3 & Pneumonia & 41 & M & 30 & Failed \\
4 & Post MVR & 68 & F & 42 & Failed \\
5 & COPD & 75 & F & 56 & Failed \\
6 & Post laparotomy & 78 & Weaned \\
7 & Post AVR & 67 & M & 22 & Weaned \\
8 & COPD & 59 & M & 14 & Weaned \\
9 & Post laparotomy & 66 & M & 20 & Weaned \\
10 & CVG & 71 & F & $<1$ & Weaned \\
11 & CVG & 61 & M & $<1$ & Weaned \\
12 & CVG & 59 & M & $<1$ & Weaned \\
13 & CVG & 66 & M & $<1$ & Weaned \\
14 & CVG & 51 & M & $<1$ & \\
\hline
\end{tabular}

COPD = chronic obstructive pulmonary disease; $M V R=$ mitral valve replacement; $A V R=$ aortic valve replacement; $\mathbf{C V G}=$ coronary vein grafts. was $5.6 \%$ (range $1 \cdot 5-14 \%$ ) and for PDI MRR was $3.0 \%(1.0-5 \cdot 5 \%)$.

MRR of POES and PDI during mechanical ventilation (control), after 15-45 minutes of unassisted ventilation and subsequent reventilation in patients who failed to wean, or after eight hours of spontaneous ventilation in patients who weaned, is given in fig 2 . In five patients weaning was unsuccessful. In these patients, during spontaneous ventilation MRR for POES and PDI fell and recovered after reventilation (ANOVA, $\mathrm{p}<0.001$ ). POES MRR fell by $47 \%$ (range $24-61 \%$ ) and PDI MRR fell by $44 \%$ (range $22-56 \%$ ). In four patients who were able to wean and were extubated and remained breathing spontaneously for a further 48 hours MRR did not alter during weaning (fig 2).

It was possible to measure $M R R$ each minute during the first six minutes of spontaneous ventilation in subject 4. POEs MRR fell each minute from 7.64 to 4.24 and PDI MRR fell from 8.2 to 4.9 (fig $3 \mathrm{~A}$ ). In subject $3 \mathrm{MRR}$ was measured each minute after reventilation. PoEs MRR rose from 4.4 in the first minute after reventilation to 9.3 after 10 minutes. PDI
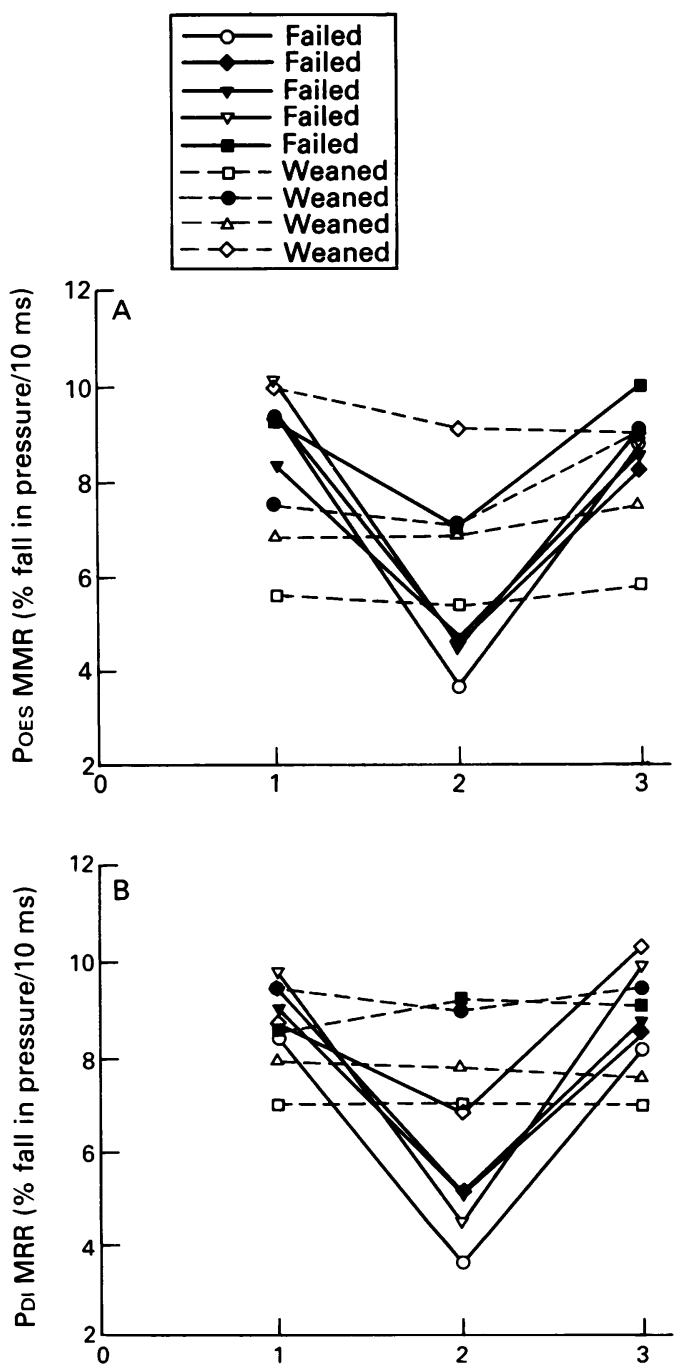

Figure 2 (A) Oesophageal and (B)

transdiaphragmatic MRR measured in nine patients weaning from mechanical ventilation. MRR was measured (1) during mechanical ventilation before weaning, (2) during spontaneous ventilation, and (3) during reventilation (failures) or after eight hours (weaned). 

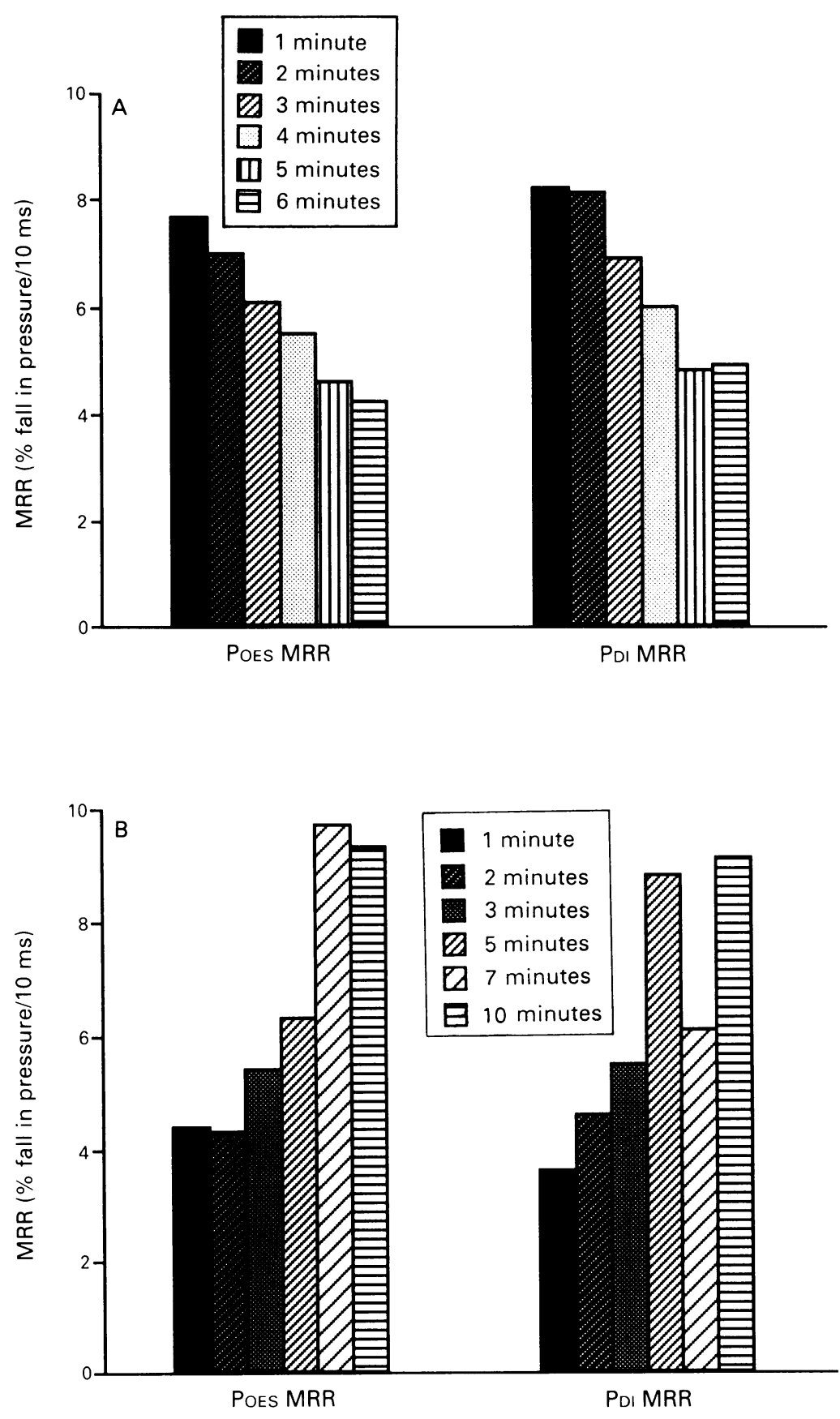

Figure 3 Oesophageal (POES) and transdiaphragmatic (PDI) MRR measured sequentially in two subjects during weaning. Figure $3 A$ shows MRR after one, two, sequentially in two subjects during weaning. Figure $3 A$ shows $M R R$ after one, two, shows MRR measured during recovery after one, two, three, five, seven, and 10 minutes of mechanical ventilation in subject 3.

MRR rose from 3.6 in the first minute to $9 \cdot 1$ after 10 minutes (fig $3 \mathrm{~B}$ ).

Figure 4 shows, for the nine patients who entered the weaning trial, peak PoEs during spontaneous breathing, maximum POEs, tidal volume, and duty cycle (TI/TTOT) over the first two minutes after cessation of mechanical ventilation, immediately before reventilation in those patients who failed to wean, and at one hour in those who weaned. In those failing to wean, peak POES per breath, TI/TTOT, and PoEsbreath/PoEsmax rose, whereas Vt tended to fall.

Clinical measurements of heart rate, respiratory rate, and respiratory distress as reported by the patient, noted at the same time periods, are given in table 2 . Only patient 1 had paradox and none had respiratory alternans.
Table 2 Clinical observations of nine patients submitted for a trial of weaning recorded (a) in the first two minutes of spontaneous breathing and $(b)$ before reventilation in those who failed to wean (1-5) or after eight hours of unassisted breathing (6-9)

\begin{tabular}{llll}
\hline Patient & Respiratory rate & Heart rate & Distress \\
\hline 1 (a) & 25 & 102 & \\
(b) & 31 & 104 & ++ \\
2 (a) & 20 & 82 & \\
(b) & 30 & 109 & ++ \\
3 (a) & 15 & 100 & \\
(b) & 25 & 99 & ++ \\
4 (a) & 31 & 92 & \\
(b) & 31 & 91 & ++ \\
5 (a) & 30 & 70 & \\
(b) & 30 & 100 & ++ \\
6 (a) & 18 & 83 & \\
(b) & 19 & 83 & - \\
7 (a) & 22 & 93 & \\
(b) & 14 & 87 & - \\
8 (a) & 25 & 79 & \\
(b) & 25 & 85 & - \\
9 (a) & 23 & 88 & \\
(b) & 23 & 92 & - \\
\hline
\end{tabular}

\section{Discussion}

This study has shown that the MRR of the inspiratory muscles can be measured from brief inspiratory efforts through a flow limiting valve, and that it falls after heavily loaded breathing in normal subjects. Using the flow limiting valve MRR can be measured in intubated patients and does not alter in patients weaned after routine surgery. In a selected group of patients recovering from severe critical illness MRR did not slow during weaning in those who succeeded in breathing spontaneously, but did slow in those patients who failed to wean and required reventilation.

MRR has not previously been measured in intubated patients. Inspiratory waveforms through the valve were closely similar to those during freely performed sniffs. Closure of the valve occurred at an inspiratory pressure of $10-14 \mathrm{~cm} \mathrm{H}_{2} \mathrm{O}$ which is similar to the pressure required to collapse the anterior nares during a freely performed sniff. ${ }^{14}$ All patients were able to perform rapid inspiratory manoeuvres without discomfort from the endotracheal tube.

At rest, POES and PDI MRR measured in our patients agreed with the values from previous studies in normal subjects ${ }^{8}$ and patients. ${ }^{9}$ The intrasubject coefficient of variation of POES and PDI MRR was $10 \%$, although $25 \%$ of subjects were not able to perform the sniff manoeuvre and were excluded, and in some of the remaining individuals only $9 \%$ of recorded sniffs could be analysed. When measured in patients MRR is more variable, with a coefficient of variation of POES MRR between $5.7 \%$ and $26 \% .{ }^{15}$ In the present study, although the coefficient of variation of POES MRR ranged between $2.4 \%$ and $20 \cdot 6 \%$, it exceeded $10 \%$ in only three patients, two of whom were in the cardiac postoperative group of patients where discomfort may have prevented the patient from performing consistent inspiratory manoeuvres.

In this study we were not able to measure simultaneously upper airway relaxation rate in the patients who were being weaned. In the laboratory the transmission of intrathoracic inspiratory waveforms can be accurately measured by balloon catheters in the mouth or nose. ${ }^{8}$ However, in patients with severe airflow 

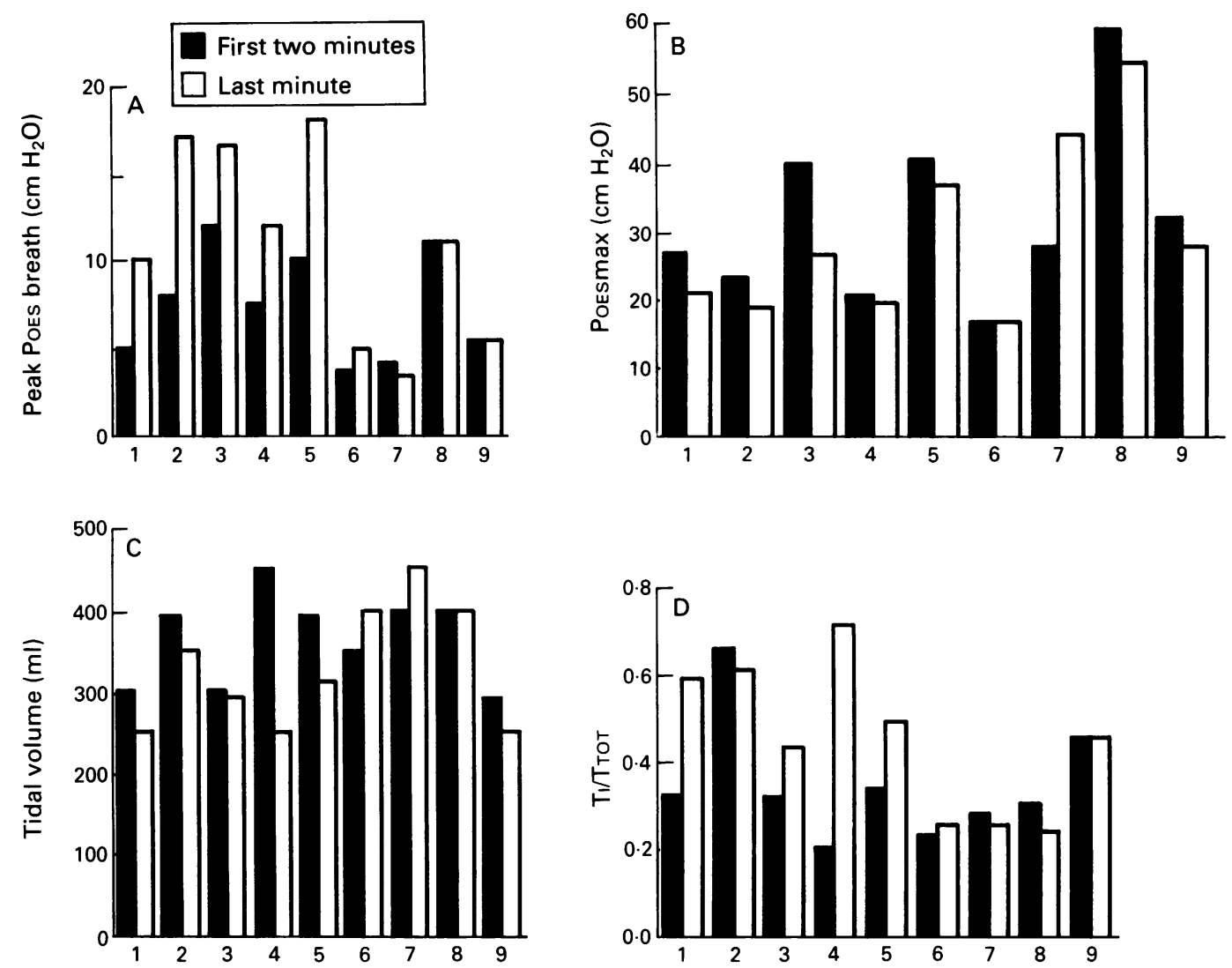

Figure 4 ( $\mathrm{A}$ ) Oesophageal pressure (POEs, $\left.\mathrm{cm} \mathrm{H}_{2} \mathrm{O}\right),(\mathrm{B})$ maximum oesophageal pressure (PoEsmax, $\left.\mathrm{cm} \mathrm{H}_{2} \mathrm{O}\right)$, (C) tidal volume (VT, ml), and (D) inspiratory duty cycle (TI/TTOT) measured within the first two minutes of spontaneous breathing and repeated either at the end of the weaning trial or after eight hours of spontaneous ventilation. Patients 1-5 failed to breathe spontaneously and were reventilated, whereas patients 6-9 were successfully weaned.

limitation or restrictive lung disease MRR may not be accurately assessed in the upper airway, ${ }^{15}$ possibly due, in part, to the differential contraction of oropharyngeal and neck muscles delaying transmission of intrathoracic pressure signals. We have shown that it is possible to measure MRR from the endotracheal tube pressures in five postoperative patients, and that the waveform is similar to that found in the oesophagus. Airway MRR data must be cautiously interpreted, but as the upper airway musculature is bypassed during endotracheal intubation, it is possible that MRR data measured from the endotracheal tube could be a faithful reflection of intrathoracic MRR.

It has been shown previously that MRR is unaltered at levels of inspiratory loading which are sustainable without fatigue, and the maximum decline in relaxation rate that occurs during resistive breathing can only be sustained for a few minutes. ${ }^{7}$ In the present study MRR fell by up to $61 \%$ with a range similar to that found previously. ${ }^{7}$ In the five patients who failed to wean, spontaneous breathing could be sustained for $\mathbf{4 5}$ minutes in one subject in whom MRR declined by the least amount, and MRR showed the greatest change in the patient with the shortest weaning trial. The percentage change of POES and PDI MRR in those patients who failed to wean correlated closely with the duration of spontaneous breathing before reventilation (POES, $R^{2}=0.94$, PDI, $R^{2}=0.92$ ).

MRR characteristically falls rapidly with severe inspiratory loading and recovers monoexponentially with rest. ${ }^{11}$ Although it was not possible to measure MRR immediately after weaning and repetitively during recovery in all patients who failed weaning, MRR did recover to baseline values in all patients after 10 minutes of controlled ventilation. Repeated estimations of MRR were possible in two patients. MRR was assessed each minute from the beginning of the weaning trial in one patient and in a second patient it was measured each minute after cessation of weaning while the patient was mechanically ventilated. During weaning MRR fell to $45 \%$ of its baseline value after five minutes and remained at this level until reventilation 20 minutes later. This finding agrees with the decline in MRR measured at high levels of loading in normal subjects, where Esau et al showed a similar rapid decline in MRR when the tension time index of the diaphragm was set at $0 \cdot 4 .^{7}$ During recovery, although not exponential, MRR increased each minute after cessation of spontaneous breathing and had recovered to the baseline value by the fifth minute.

Is it possible that the change in MRR measured during spontaneous breathing in those patients who failed to wean is related to factors other than heavy loading? The effect of lung volume on POES and PDI MRR has been investigated by Levy and coworkers. ${ }^{9} \mathrm{Al}-$ though MRR can be increased (from a mean value of 7.6 to 8.4 ) by increasing lung volume, it is not reduced by reducing lung volume from functional residual capacity. In order to standardise the end expiratory point in our 

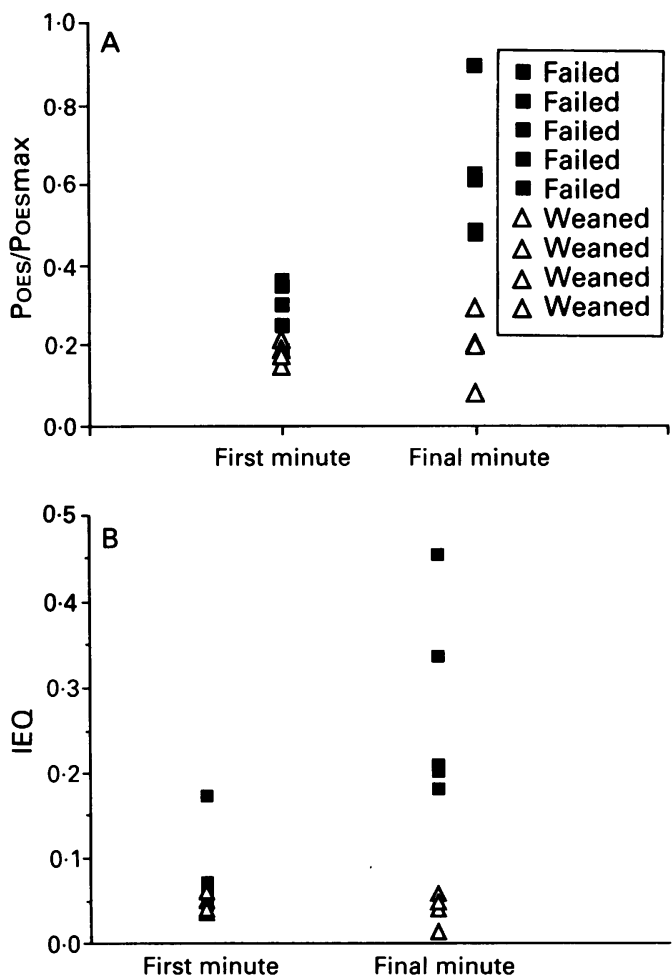

Figure 5 (A) POES/PoEsmax and (B) inspiratory effort quotient (IEQ) measured after one minute of spontaneous breathing and then at reinstitution of mechanical ventilation or after eight hours of spontaneous breathing in nine patients weaning.

patients, measurements were made from a resting end expiratory pressure that varied by only $1-2 \mathrm{~cm} \mathrm{H}_{2} \mathrm{O}$ between efforts, they were made in the same posture, and were qualitatively compared with previous efforts with a respiratory inductive plethysmograph. In addition, the changes seen in the present study are of a magnitude hitherto seen only during excessive inspiratory resistive loading. It is therefore unlikely that the decrease in MRR seen in our patients could be ascribed to changes in lung volume or the length of the respiratory muscles.

The relation between load and respiratory muscle strength can be quantified from the ratio of pressure generated per breath to maximum pressure (PoEs/PoEsmax), ${ }^{4}$ the tension time index of the diaphragm (TTDI, PDI/PDI$\max \times$ TI/TTOT), ${ }^{6}$ and the inspiratory effort quotient (IEQ, $(k \times V T / C d y n) \times(T I / T T O T) /$ Pmax). ${ }^{16}$ Normal subjects are unable to sustain oesophageal pressures which exceed $40 \%$ of maximum or a TTDI greater than $15 \%$ of maximum, and in patients an IEQ of 0.19 or more is associated with weaning failure. ${ }^{17}$ To confirm that the changes seen in MRR in this study occurred during extreme loads, we measured peak oesophageal pressure, the duty cycle, and maximum oesophageal pressure during weaning. Successfully weaned patients had a smaller oesophageal pressure in relation to maximum pressure (POEs/PoEsmax) when compared with those patients who were reventilated, and IEQ exceeded 0.19 in all patients who failed to wean (fig 5). This suggests that, in our patients, when the load applied to the respiratory muscles exceeded their capacity or strength, weaning failure occurred and was associated with a slowing of MRR.

The importance of the dynamic balance between load and strength is illustrated by individual patients within our series. Profound weakness was associated with weaning success only when oesophageal pressure per breath was low, and therefore PoEs/PoEsmax and IEQ were less than the threshold value for sustainable ventilation (patient 6, PoEsmax $=17$, PoEs $/$ PoEsmax $=0.21$ and IEQ $=0.05$ ). This contrasts with patient 2 with equally low maximal pressures, but a greater load applied to the respiratory muscles.

We conclude that MRR can be measured in intubated patients in intensive care and has a numerical range and variability similar to that previously recorded by other workers in nonintubated patients. MRR declined only in those patients who failed to wean from mechanical ventilation, and recovered with rest when patients were reventilated. A fall in MRR was associated with heavily loaded respiratory muscles, although it was the balance between load and strength that was of critical importance. Further studies are required to determine the clinical usefulness of this technique, but since MRR slows before maximum force is reduced, it may be a suitable tool for detecting those patients who will fail to wean at the beginning of trials of spontaneous breathing.

1 Murciano D, Boczkowski J, Lecocguic Y, Milic-Emili J, Pariente R, Aubier M. Tracheal occlusion pressure: a simple index to monitor respiratory muscle fatigue during acute respiratory failure in patients with chronic obstructive pulmonary disease. Ann Intern Med 1988;108:800-5.

2 Montgomery AB, Holle RHO, Neagley SR, Pierson DJ, Schoene RB. Prediction of successful ventilator weaning using airway occlusion pressure and hypercapnic challenge. Chest 1987;91:496-9.

3 NHLBI Workshop summary. Respiratory muscle fatigue. Report of the Respiratory Muscle Fatigue Workshop Group. Am Rev Respir Dis 1990;142:474-80.

4 Roussos CS, Macklem PT. Diaphragmatic fatigue in man. $f$ Appl Physiol 1977;43:189-97.

5 Bellemare F, Grassino A. Evaluation of human diaphragm fatigue. F Appl Physiol 1982;53:1196-206.

6 - Bellemare F, Grassino A. Effect of pressure and timing of contraction on human diaphragm fatigue. 7 Appl Physio 1982;53:1190-5.

7 Esau SA, Bellemare F, Grassino A, Permutt S, Roussos C, Pardy RL. Changes in relaxation rate with diaphragmatic fatigue in humans. $\mathcal{F}$ Appl Physiol 1983;54:1353-60.

8 Koulouris N, Vianna LG, Mulvey D, Green M, Moxham J. Maximal relaxation rate of esophageal, nose and mouth pressures during a sniff reflect inspiratory muscle fatigue. Am Rev Respir Dis 1989;139:1213-7.

9 Levy RD, Esau SA, Bye PTP, Pardy RL. Relaxation rate of mouth pressures with sniffs at rest and with inspiratory
muscle fatigue. Am Rev Respir Dis 1984;130:38-41.

10 Wiles CM, Young A, Jones DA, Edwards RHT. Relaxation rate of constituent muscle-fibre types in human quadriceps. Clin Sci 1979;56:47-52.

11 Jones DA. Muscle fatigue due to changes beyond the neuromuscular junction. In: Human muscle fatigue. physiological mechanisms (Ciba Foundation Symposium 82). London: Pitman Medical, 1981:178-96.

12 Milic-Emili J, Mead J, Turner JM, Glauser EM. Improved technique for estimating pleural pressure from esophageal balloons. $\mathcal{F}$ Appl Physiol 1964;19:207-11.

13 Bridger GP. Physiology of the nasal valve. Arch Otolaryngo 1970;92:543-53.

14 Pertuze JJ, Lim TK, Watson A, Pride NB. Transdiaphragmatic pressures during static and dynamic maximum inspiratory efforts in normal subjects (abstract). Bull Eur Physiopathol Respir 1987; Suppl 12:355S.

15 Mulvey DA, Elliott MW, Koulouris NG, Carroll MP Moxham J, Green M. Sniff esophageal and nasopharynMeal pressures and maximal relaxation rates in patients geal pressures and maximal relaxation rates in patients with respiratory

16 Milic-Emili J. Is weaning an art or a science? Am Rev Respir Dis 1986;134:1107-8.

17 Gandia F, Blanco J. Evaluation of indexes predicting the outcome of ventilator weaning and value of adding outcome of ventilator weaning and value of adding 1992;18:327-33. 